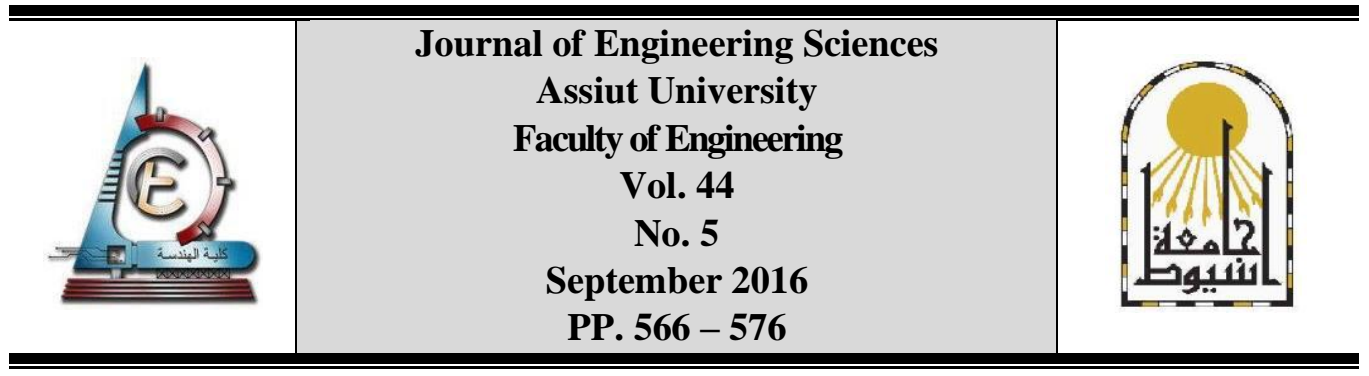

\title{
EXPERIMENTAL STUDY FOR DISPERSION AND ATTENUATION CORRECTION IN VISCOELASTIC SPLIT HOPKINSON PRESSURE BAR ARRANGEMENT
}

\author{
Reda M. Othman ${ }^{1}$, Wael M. Khier ${ }^{2}$, Abu-bakr A. Naser ${ }^{3}$ \\ Mechanical Engineering Department, Faculty of Engineering, Assiut University
}

Received 3 August 2016; Accepted 29 August 2016

\begin{abstract}
Split Hopkinson pressure Bar (SHPB) arrangement is the most practical and accurate method for studying dynamic mechanical behavior of materials under high strain rate. However in case of studying foams, and soft tissues (low impedance materials) a modified version of SHPB with bars made from a viscoelastic material like Poly methyl methacrylate PMMA acrylate is commonly used so a good prediction of the stress waves and particle velocity acting at the interface between bars and specimen taking into account the dispersion and attenuation effect is essential. In this study the wave propagation coefficient as a function of the phase velocity and the attenuation coefficient in relation to the frequency are calculated experimentally by a set of free end bar tests using a cylindrical projectile to the circumstances of stepped velocity and different firing pressure. Correction processes for longitudinal stains related to incident and reflected waves are preceded in frequency domain using Fast Fourier Transformer (FFT) technique.
\end{abstract}

Keyword: Split Hopkinson pressure Bar (SHPB), viscoelastic, dispersion, attenuation coefficient

\section{Introduction}

Characterization of the mechanical behavior of biological materials especially human body under dynamic loading conditions is essential like automotive crashes, ballistic impact, and violent sports[1].Split Hopkinson pressure bar (SHPB) arrangement can be used in testing material at strain rate ranging from $100 \mathrm{~s}^{-1}$ to $1,000 \mathrm{~s}^{-1}$.In fig.1 a schematic drawing showing SHPB configuration, which consist of three uniform cylindrical bars namely the striker, incident and transmitted bar. The specimen usually placed between the incident and transmitted bars. The striker bar is fired using a controlled pressured air toward the incident bar generating a compression stress wave which travel along the incident to the specimen interface. Portion of the wave transmitted to the specimen while the remaining reflect back to the relived incident bar[2]. Once specimen is loaded, part of the loading wave propagates to the transmitted bar while the difference consumed within the specimen. The strain gauge stations attached to the middle of the incident and transmitted bars record three strain waves signals namely $\varepsilon_{\mathrm{I}}(t), \varepsilon_{\mathrm{r}}(t)$ and $\varepsilon_{\mathrm{t}}(t)$ which are the incident, reflected and transmitted strain waves respectively[3]. In classic SHPB the bars are made from an elastic material commonly steel which have high impedance so it fails to give a correct results in case of soft tissues or foam materials which has a low impedance characteristics. 
Since it was proved that to improve the transmitted signal, the mechanical impedance of the bars material values must approximately match the tested material[4]. A modified SHPB with viscoelastic bars was suggested to give more accurate results. But as a result of viscoelasticity nature of the bars, the shape of the propagated wave change as it propagates along the bars. This mean that the force and particle velocity at the interface of the bar and the specimen differ from the signal recorded at the midst of the bar, so a correction technique of the wave's histories must be used to determine the signal at any position along the bar depending on the properties of the bars materials. Parameters like such as complex modules of elasticity $\check{E}(\omega)$, wave propagation coefficient $\mathrm{\gamma}(\omega)$, phase velocity $\alpha(\omega)$, and wave number $\mathrm{K}(\omega)$ must be calculated.

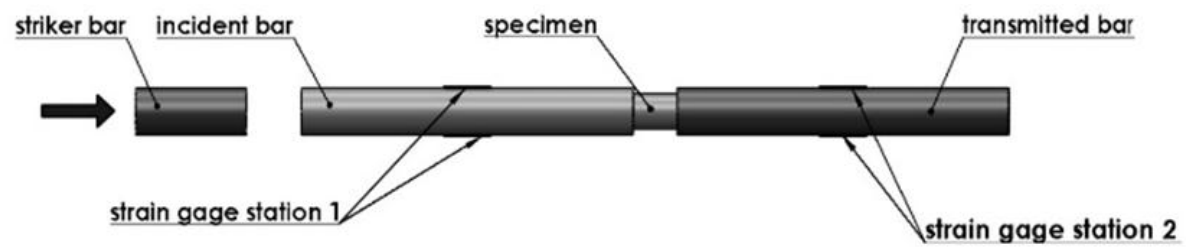

Fig. 1. Schematic drawing showing SHPB apparature

There are several experimental or mathematical methods have been investigated for this purpose, in 1994 Wang et al generalized a SHPB technique by using modified polymeric bars. They determined the stresses and particle velocities at two interfaces of specimen and the two bars based on the Zhu-Wang-Tang viscoelastic constitutive equation[5], and so In 1997 Zhao and Gary presented but the study was based on the three-dimensional Fourier harmonic wave analysis[6]. In an extended study from 2007to 2015[7][8][9][10]they determined the material properties of the PMMA bars of SHPB using different experimental methods and at different rates. M. Aleyaasein used models which may take only 2 models which compose of groups of springs and dashpots as in fig (2) where Ea and Em are the Young moduli of the purely elastic part and the Maxwell part respectively, and the parameter $\theta$ is defined by $\eta$ the viscoelastic damping constant[11].
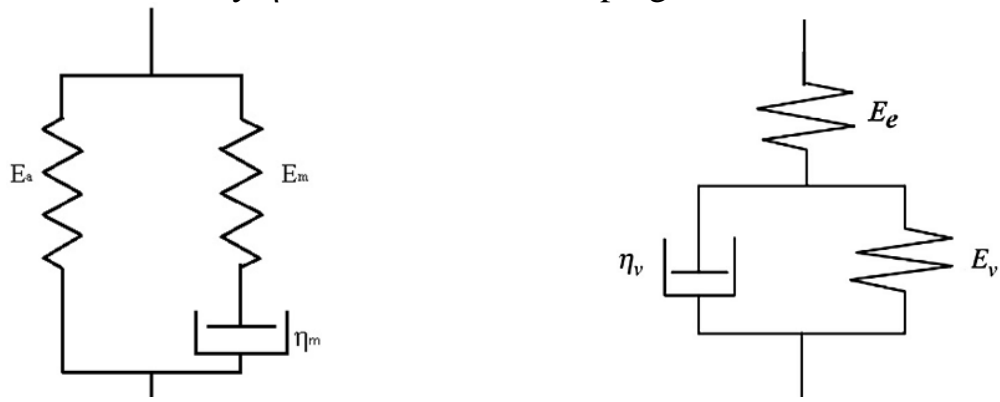

Fig. 2. standard linear solid model (SLS) model, (a) Elastic spring in parallel with a Maxwell element (b) Elastic spring in series with a KelvineViogt element.

$$
\eta=\theta E_{m}
$$

The main idea of the analytical study of the visco-bars parameter in [12],[13],[4],[14], and [15] depend on finding the mathematical relations between the wave number, attenuation coefficient, wave propagation coefficient, and complex modulus, as in equs (2,3). This analytical solutions is available only in case of knowing all the frequency dependent material parameters of the bar material. In advanced experimental test, it is inaccurate to generalize properties for such materials as these are dependent on the loading rate, environmental history, and manufacturing processconditions[9]. 


$$
\begin{aligned}
& \alpha^{2}=\frac{\rho \omega^{2}}{2 E_{a}}\left(\sqrt{\frac{1+\omega^{2} \theta^{2}}{\left(1+\frac{E_{m}}{E_{a}}\right)^{2} \omega^{2} \theta^{2}+1}}-\frac{\left(1+\frac{E_{m}}{E_{a}}\right) \omega^{2} \theta^{2}+1}{\left(1+\frac{E_{m}}{E_{a}}\right)^{2} \omega^{2} \theta^{2}+1}\right) \\
& k^{2}=\frac{\rho \omega^{2}}{2 E_{a}}\left(\sqrt{\frac{1+\omega^{2} \theta^{2}}{\left(1+\frac{E_{m}}{E_{a}}\right)^{2} \omega^{2} \theta^{2}+1}}+\frac{\left(1+\frac{E_{m}}{E_{a}}\right) \omega^{2} \theta^{2}+1}{\left(1+\frac{E_{m}}{E_{a}}\right)^{2} \omega^{2} \theta^{2}+1}\right)
\end{aligned}
$$

In all previous experimental work a steel ball striker projectile was used and that doesn't simulate the realistic set of the arrangement which the striker is a cylinder with the same material and diameter of the other two bars, So in this paper an experimental study of SHPB with viscoelastic bars using cylindrical strikers at different pressures and velocities to identify the wave propagation coefficient by designation the wave number and attenuation coefficient, then correct the measured strain and determine the contact force. The correction technique is used at the frequency domain by applying The Fast Fourier transforms to the collected incident and reflected signals.

\section{Theoretical considerations}

The classical SHPB arrangement uses the one dimension wave propagation theory, and the propagated waves through the bars have negligible effect; therefore, the measured strains by the gauges at the middle of the bars can be the same at the specimen-bar interfaces so strains of three waves can be expressed as $\varepsilon_{\mathrm{I}}(t), \varepsilon_{\mathrm{r}}(t)$ and $\varepsilon_{\mathrm{t}}(t)$. The force and particle velocity at the end of each bar can be delivered from equs (4-7)

$$
\begin{gathered}
F_{\text {in }}=A E\left[\varepsilon_{I}(t)+\varepsilon_{R}(t)\right] \\
F_{\text {out }}=A E\left[\varepsilon_{T}(t)\right] \\
V_{\text {in }}=C E\left[\varepsilon_{I}(t)-\varepsilon_{R}(t)\right] \\
V_{\text {out }}=C E\left[\varepsilon_{T}(t)\right]
\end{gathered}
$$

Where, A is the cross-sectional area of the bars, E the Young's modulus of the material, and $\mathrm{C}$ the stress wave velocity. as explained in case of dynamic testing of soft low mechanical impedance materials, the classical SHPB method is inaccurate, from Eqn. (4)-(7) it is clear that, the accuracy of the output force and velocity depend on the accuracy of the measuring strains delivered to the specimen by the transmitted wave. Moreover, the input force and velocity cannot be determined, if the mechanical impedance of the specimen is very small than the pressure bars. It is found that the transmitted pulse is very low in magnitude due to reflection of the majority of incident wave back into the input bar, so the input force tends to be zero. It is essential to use low impedance pressure bars for the accuracy of the tests and so the steel bars in classical SHPB werereplaced byPMMA low impedance bars. Due to the viscoelastic characteristic of the material, the wave dispersion and attenuation effects are increased. To correct this effect the accurate method is to predict the wave at any position along the bar by determination of the wave propagation coefficient ( $\mathrm{\gamma}$ ).In the frequency domain, the general relationship for a longitudinal wave propagating along a rod is: 


$$
\varepsilon^{\sim}(x, \omega)=P^{\sim}(x, \omega) e^{-\gamma^{\sim}(\omega) d}+N^{\sim}(x, \omega) e^{\gamma^{\sim}(\omega) d}
$$

Where $\omega$ is circular frequency, $\mathrm{P}^{\sim}$ and $\mathrm{N}^{\sim}$ are the Fourier transformation (FT) of the incident and reflected strains respectively at $\mathrm{x}=0 . \mathrm{\gamma}^{\sim}(\omega)$ is the propagation coefficient defined as:

$$
\tilde{\gamma}(\omega)=\alpha(\omega)+i k(\omega)=\alpha(\omega)+i \frac{\omega}{C(\omega)}
$$

Where $\alpha(\omega)$ is the attenuation coefficient and $\mathrm{k}(\omega)$ is the wave number. The attenuation coefficient refer to the reduction of magnitude of a propagating wave while the wave number is related to the phase velocity $C(\omega)$ and refer to the dispersion of waves of different frequencies.

To find the Fourier transforms of the axial particle velocity $\tilde{V}$ and the normal forceF ${ }^{\sim}$ at any cross section $\mathrm{x}$ :

$$
\begin{gathered}
\tilde{V}(x, \omega)=\frac{-i \omega}{\gamma(\omega)}\left[P^{\sim}(x, \omega) e^{-\gamma^{\sim}(\omega) d}-N^{\sim}(x, \omega) e^{\gamma^{\sim}(\omega) d}\right] \\
F^{\sim}(x, \omega)=\frac{-\rho A \omega^{2}}{\gamma^{2}(\omega)}\left[P^{\sim}(x, \omega) e^{-\gamma^{\sim}(\omega) d}+N^{\sim}(x, \omega) e^{\gamma^{\sim}(\omega) d}\right]
\end{gathered}
$$

To get the force, velocity, and strains use the invers of the FFT of the results in time domain. The complex Young's modulus of the viscoelastic material Ě. Found by:

$$
\check{\mathrm{E}}=-\frac{\rho \omega^{2}}{\gamma^{2}(\omega)}=\dot{\mathrm{E}}(\omega)+i \grave{\mathrm{E}}(\omega)
$$

Its real and imaginary parts correspond to storage and loss moduli, É and Ë respectively.

\section{Experimental set up}

To determine the wave propagation coefficient, the test set up prepared as shown in the figure (3). The three cylindrical bars striker (St), incident(Inc), and transmitted(Tr) are the same diameter $40 \mathrm{~mm}$, and made of an extruded PMMA acrylate with density $\rho=1190 \mathrm{~kg} / \mathrm{m}^{3}$. Inc and Tr bars are $2 \mathrm{~m}$ in length. Both Inc and Tr bars kept aligned using (Poly tetrafluoro ethylene) PTTF bearing. Data of the travelling waves collected and monitoring by two strain gage station at the middle of the bars, the gages are connected to an amplifier designed and manufactured to suit this high rate of loading then to aTektronix Digital oscilloscope. Two active strain gages are glued in opposite exact positions in the middle of bar as shown in figure (3).Cylindrical St bar was used with length (34mm)fired to the direction of the Inc bar at a predefined speeds. The velocity of St bar was correlated to the pressure in the pneumatic mechanism and recorded using 2 PNB laser sensors.

Five tests at different pressures were performed to evaluate the propagation coefficient at different impact velocities i.e. at different levels of excitations. The test at each pressure was repeated twice, resulting in total 10free end tests as in table (1). 


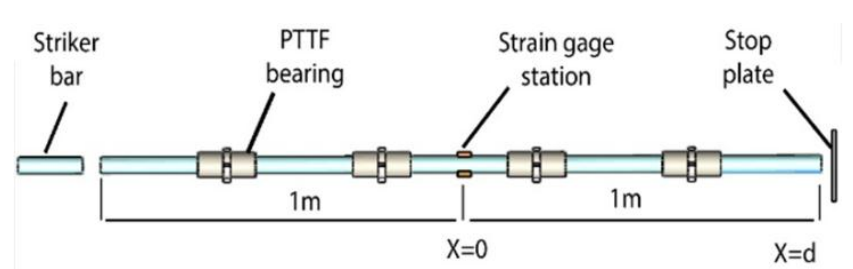

Fig. 3. SHPB arrangement for free end test

Table 1.

Test parameters for determination of the propagation coefficient

\begin{tabular}{|c|c|c|}
\hline Test No. & Pressure (bar) & Velocity $(\mathrm{m} / \mathrm{s})$ \\
\hline 1 & 0.5 & 11 \\
\hline 2 & 0.6 & 15 \\
\hline 3 & 0.7 & 20 \\
\hline 4 & 1 & 27 \\
\hline 5 & 1.5 & 33 \\
\hline
\end{tabular}

\section{Calculation principle}

Assuming a finite bar with length $(\mathrm{L}=2 \mathrm{~d})$ is impacted at one end and the other is free end. The strain gage is centered at the midst where $x=0$, and the free end is at $x=d$ as in fig.(3). The generated incident and reflected waves is recorded separately. The striker bar must be short enough to prevent wave signals overlap (one tenth of the incident bar length at most). The force component at the free end at $(\mathrm{x}=\mathrm{d})$ must be equal to zero, then eqn. (11) now become:

$$
\left[P^{\sim}(0, \omega) e^{-\gamma^{\sim}(\omega) d}+N^{\sim}(0, \omega) e^{\gamma^{\sim}(\omega) d}\right]=0
$$

As a result, the transfer function $\mathrm{G}(\omega)$ is given by

$$
\begin{gathered}
G(\omega)=-\frac{\tilde{\varepsilon_{R}}(0, \omega)}{\tilde{\varepsilon}_{I}(0, \omega)}=e^{\gamma(\omega) 2 d} \\
\alpha(\omega)=-\ln \left(\frac{a b s \tilde{\varepsilon^{\sim}}(0, \omega)}{a b s \tilde{\varepsilon}_{I}(0, \omega)}\right) / 2 d \\
K(\omega)=-\left(\frac{\theta_{R-} \theta_{I}}{2 d}\right) \\
C(\omega)=\frac{\omega}{K(\omega)}
\end{gathered}
$$

Through investigation of the transfer function, the propagation coefficient is calculated. The attenuation coefficient $\alpha(\omega)$ is easily delivered from the amplitude of the transfer function as in eq.(15), then the wave number $K(\omega)$ is obtained from the phase the transfer function as in eq.(16). But the phase spectra must be subjected to the numerical procedure known as unwrapping to correct the radian phase angles in a vector $\mathrm{P}$ by adding multiples of $\pm 2 \pi$ when absolute jumps between consecutive elements of $\mathrm{P}$ are greater than or equal to the default jump tolerance of $\pi$. Then, the wave number will be described by a real number. 


\section{Results}

The data collected from strain gage stations readings refer to the waves signal recorded at the middle of the bars, can be separated into two signals $\varepsilon_{\mathrm{I}}(t), \varepsilon_{\mathrm{r}}(t)$ as shown in Figure 4. (A). The figure illustrates the raw data acquired from the free end test performed on one of the acrylic bars. The raw data signal is comprised of two parts. The first pulse indicated is the passage of the incident wave by the strain gauge. This wave is generated by the impact of the striker and travels along the length of the bar towards the interface end. The inverted second pulse indicated is the return passage (reflection) of the incident wave off the free end. After Comparing incident and reflected waves it is obviously the attenuation and dispersion effects which can be caused by a variety of factors such as, material properties and geometric constraints as in fig.4.(B)

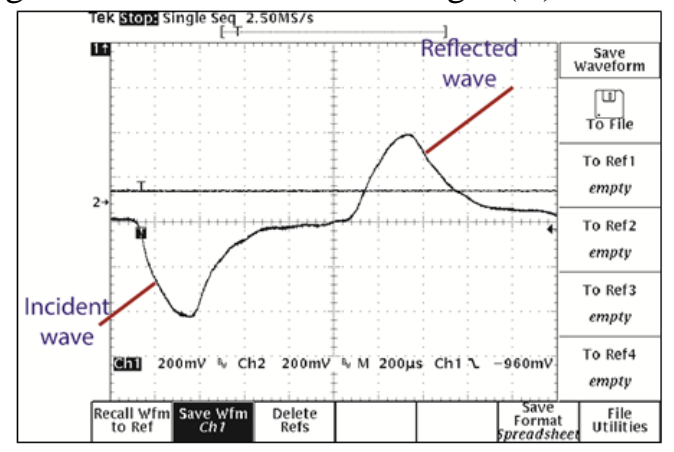

(A)

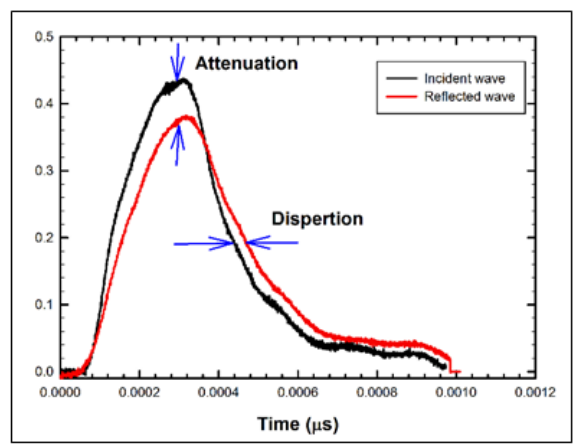

(B)

Fig. 4. ( A ) The typical oscilloscope output signal after the free end bar test, only one strain gage station reading,(B) Illustration of the Effects of Dispersion and Attenuation on reflected wave compare to incident wave.

\subsection{Wave propagation parameters calculation}

Depending on Excel and Matlab code, wave propagation parameters were carried out. The signal data were collected in spread sheet file with 5000 point captured within $2 \mathrm{msec}$ after each test. All these points were defined the incident wave and its reflection, then the two mentioned waves $-\mathcal{E} I$ and $\mathcal{E}$-separated into two double arrays, which increased to 8192 by adding a series of zeros to the initial 2500 recorded points for each. Thus, the calculated parameters phase velocity and the attenuation coefficient versus frequency curves becomes smoother. After taking the FFT of the axial strains, $\mathcal{E}$ I and $\mathcal{E R}$, obtained the values of $\tilde{\varepsilon_{I}}(\omega)$ and $\tilde{\varepsilon_{R}}(\omega)$ within the same periodic time from zero to 1000 microseconds with step of $0.4 \mu \mathrm{sec}$. At this way the definition of the transfer function $\mathrm{G}(\omega)$ was obtained easily, see Eq. (14).

To calculate the attenuation coefficient and phase velocity, the waves (incident and reflected) were then transformed into the frequency domain, Recall from equation (15) that the attenuation coefficient is a function of the ratio of the amplitude spectra of the incident and reflected pulses. Similarly, a difference in phase spectra for the incident and reflected waves can be related to the wave number (through equation (16)) and corresponding phase velocity as in equation (17), the phase of the each strain Inc and Re is determined in the range between 0 and $2 \pi$ Therefore, Once these two parameter are calculated, the wave propagation coefficient now can be easily delivered as in equation (9). Then the two main 
parameters were plotted in to fig (5), and so as to the two parameters of complex modulus of elasticity were calculated using eq.11 and plotted in fig (7).

It can be noted that these results figure (5,and 6) are quite mismatch up toabout5 $\mathrm{kHz}$. Above this frequency, the scattering increases because the signal spectrum components become lower. That is due to the pulse duration which is depending on the projectile diameter, and the frequency range of the measurements. Short pulses associate with small diameter projectiles, this way the accuracy of the measuring technique would be increase. The accurate frequency range reported $0-5 \mathrm{kHz}$, for suitably accurate results for projectile diameters larger than $12 \mathrm{~mm}$ and up to $40 \mathrm{~mm}[10]$
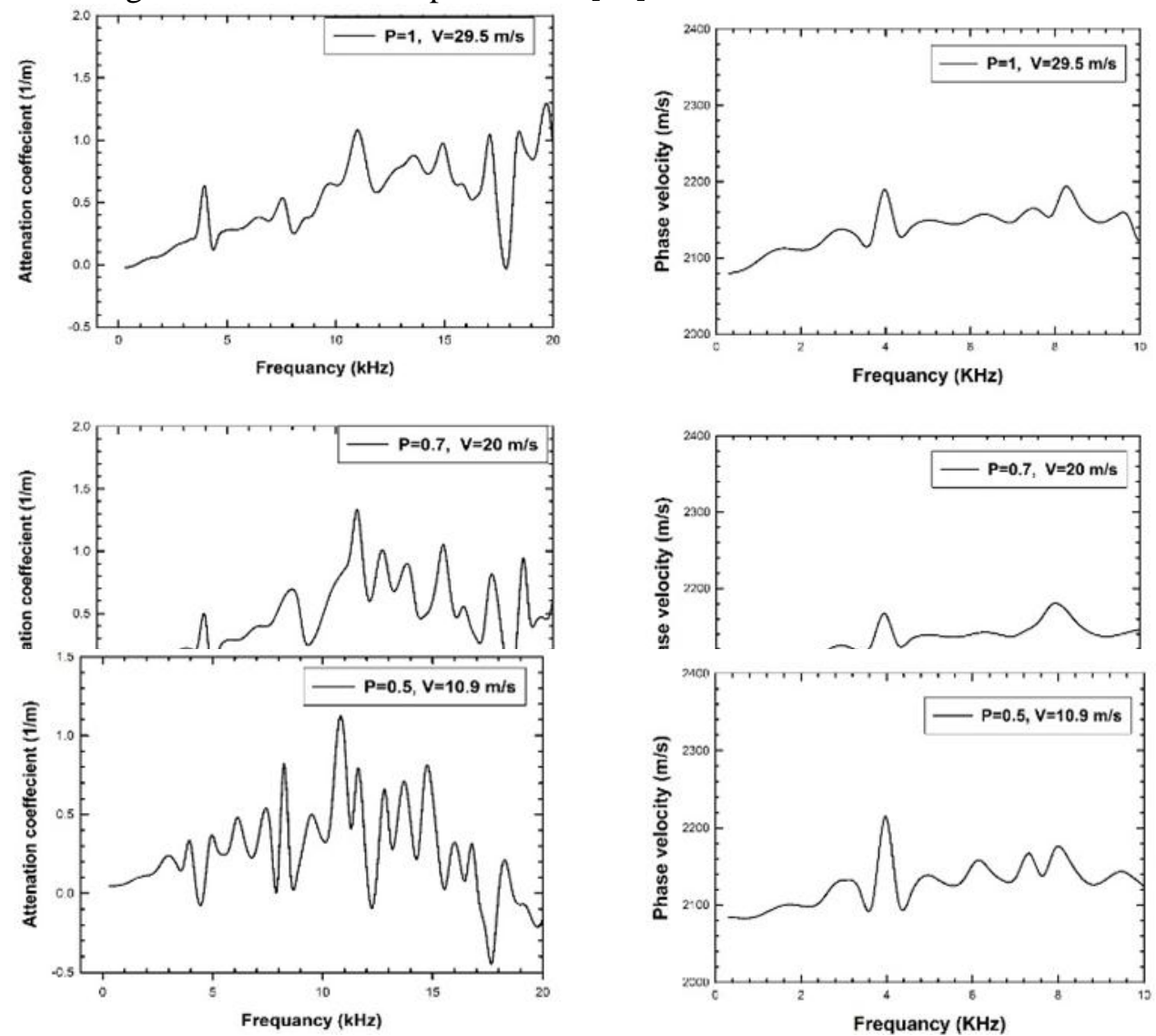

Fig. 5. Attenation coeffecient and phase velocity for defferent test parameter
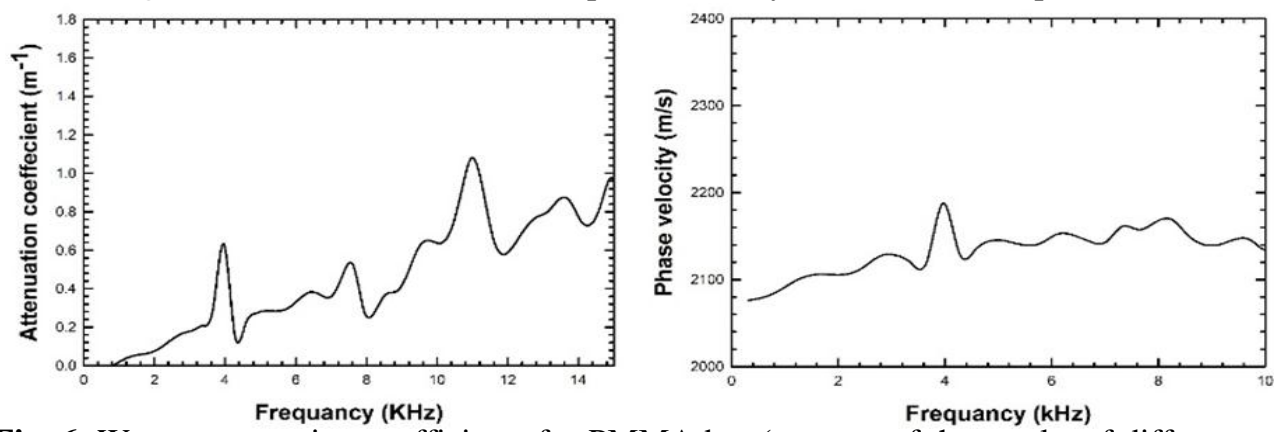

Fig. 6. Wave propagation coefficients for PMMA bar (average of the results of different striker impacts) (a) attenuation coefficient (b) phase velocity 


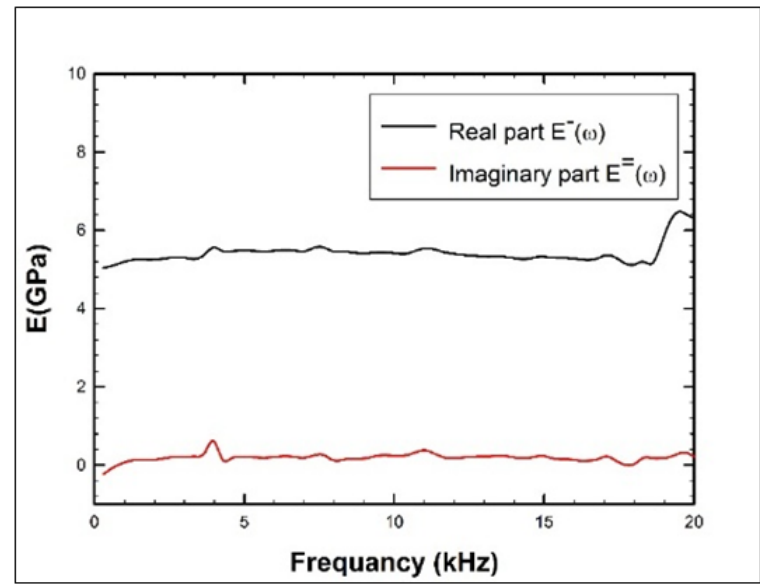

Fig. 7. Average values of Complex modulus $E$ of the PMMA versus frequency

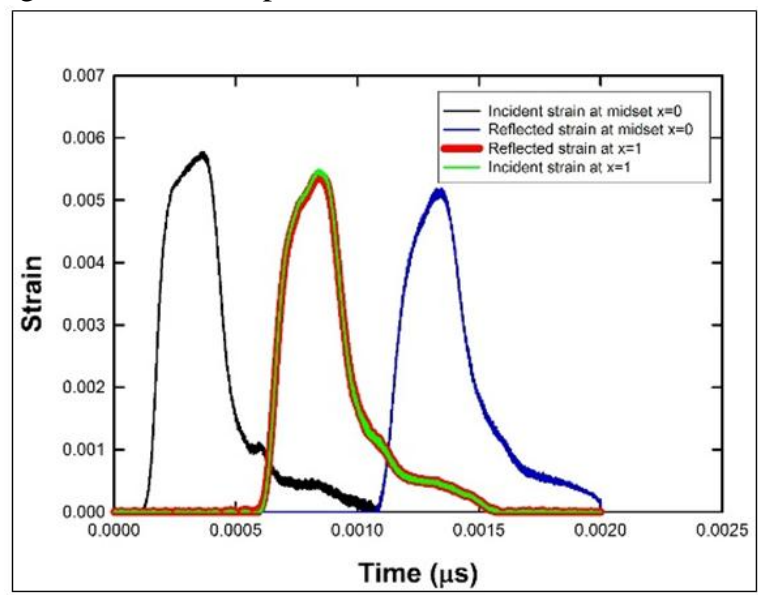

Fig. 8. Strain befor and after correction

\subsection{Results validation}

For results validation a comparison between the measured and predicted waves were undertaken. The wave induced at middle of the bar (incident wave which capturing by the strain gage station) propagate through the bar toward the free end then reflect back, the strain gage station record it as a reflected wave. So if the predicted wave forward to the free end from the incident one is match the other predicted wave back to the free end from the reflected wave, all the parameter used in prediction is certainly correct, that is clear in figure (8). That is because of the difference of the material properties at the end of the bar which became air and thus the entire wave at this end reflect back passing through strain gage station again. That is mean the difference between the two waves predicted at the end of the bar must refer to zero.

\subsection{Correction procedures}

Assuming that the wave propagate through the bar and measured by the strain gage station at the middle length of the bar is $\mathrm{U}_{0}$, to calculate this wave at any position on the bar length, some steps must be done. 
- FFT is computed for the digitized wave signal collected from strain gage station and gain its frequency spectrum.

- Attenuation factor is identified and phase velocity for each frequency component of the wave using Equs (15) or (17), and (9).

- The waves at $x=0$ needs to be corrected or predicted from the measured wave at the position of $\mathrm{x}=\mathrm{d}$. Construct the frequency spectrum of the wave to be corrected or predicted using the following equation

$$
\begin{gathered}
\tilde{\varepsilon^{\sim}}(0, \omega)=\operatorname{FFT}\{\varepsilon(0, t)\} \\
\tilde{\varepsilon^{\sim}}(d, \omega)=\varepsilon^{\tilde{\varepsilon}}(0, \omega) \cdot e^{\gamma d}=\varepsilon^{\sim}(0, \omega) \cdot e^{(\alpha+i k) d}
\end{gathered}
$$

- The predicted or corrected waves signals are obtained using the Inverse Fast Fourier Transform IFFT

$$
\varepsilon(d, \omega)=\operatorname{IFFT}\left\{\varepsilon^{\sim}(d, \omega)\right\}
$$

\section{Conclusion}

- Modified Split Hopkinson Pressure Bar arrangement with viscoelastic bars was found suitable to test soft materials with low impedance characteristics. Due to viscoelastic effect of the bars, the measured strains at the midpoint of the bars are differ from the actual strains induced to specimen, so calculating wave propagation factor of the bars material is important step to measure strain at any point along the bar. An experimental method is delivered for measuring wave propagation factor with regards to dispersion and attenuation effect. Using cylindrical striker to free end PMMA bar test set, with applying FFT to the longitudinal strain waves and it's reflected the propagation coefficient, phase velocity and attenuation coefficient were determined. All parameters expressed as monotonic functions of the frequency. The main remarks of the results informed that:

- The accuracy of this technique strongly depends on the duration of the pulse which is associated with the diameter and length of the striker bar, and the frequency range of the measured data.

- The scattering of the propagation coefficient due to different impact velocity can be reduced by averaging the results.

\section{REFERENCES}

[1] M. M. Trexler, a M. Lennon, a C. Wickwire, T. P. Harrigan, Q. T. Luong, J. L. Graham, a J. Maisano, J. C. Roberts, and a C. Merkle, "Verification and implementation of a modified split Hopkinson pressure bar technique for characterizing biological tissue and soft biosimulant materials under dynamic shear loading.," J. Mech. Behav. Biomed. Mater., vol. 4, no. 8, pp. 1920-8, Nov. 2011.

[2] C. Hernandez, a. Maranon, I. a. Ashcroft, and J. P. Casas-Rodriguez, “An inverse problem for the characterization of dynamic material model parameters from a single SHPB test," Procedia Eng., vol. 10, pp. 1603-1608, 2011.

[3] E. Palamidi, "Hopkinson Bar testing of cellular Materials," The University of Manchester, 2010.

[4] H. Zhao, "A three dimensional analytical solution of the longitudinal wave propagation in an infinite linear viscoelastic cylindrical bar. Application to experimental techniques," J. Mech. Phys. Solids, vol. 43, no. 8, pp. 1335-1348, 1995.

[5] L. Wang, K. Labibes, z. Azari, and G. Pluvinage, "Generalization of split Hopkinson bar technique to use viscoelastic bars,” Int. J. Impact Eng., vol. 16, no. 3, pp. 529-530, 1994. 
[6] H. Zhao, G. Gary, and J. Klepaczko, "On the use of a viscoelastic split hopkinson pressure bar,” Int. J. Impact Eng., vol. 19, no. 4, pp. 319-330, 1997.

[7] C. Salisbury, "Spectral Analysis of Wave Propagation Through a Polymeric Hopkinson Bar," University of Waterloo, 2001.

[8] S. Mousavi, "Identification of Viscoelastic Materials by Use of Wave Propagation Methods," Uppsala University, Sweden, 2007.

[9] H. S. U. Butt and P. Xue, "Determination of the wave propagation coefficient of viscoelastic SHPB: Significance for characterization of cellular materials,” Int. J. Impact Eng., pp. 1-9, 2013.

[10] I. D. Theodorakopoulos, "Determination of the Useful Frequency for the Calculation of the Attenuation Coefficient and Phase Velocity in a Viscoelastic Hopkinson Pressure Bar," Int. Appl. Mech., vol. 51, no. 3, pp. 360-368, 2015.

[11] M. Aleyaasin and J. J. Harrigan, "Wave dispersion and attenuation in viscoelastic polymeric bars: Analysing the effect of lateral inertia," Int. J. Mech. Sci., vol. 52, no. 5, pp. 754-757, 2010.

[12] E. D. H. Davies and S. C. Hunter, "The dynamic compression testing of solids by the method of the split Hopkinson pressure bar," J. Mech. Phys. Solids, vol. 11, no. 3, pp. 155$179,1963$.

[13] J. Z. Malinowski and J. R. Klepaczko, "A unified analytic and numerical approach to specimen behaviour in the Split-Hopkinson pressure bar," Int. J. Mech. Sci., vol. 28, no. 6, pp. 381-391, 1986.

[14] a. Benatar, D. Rittel, and a. L. Yarin, "Theoretical and experimental analysis of longitudinal wave propagation in cylindrical viscoelastic rods," J. Mech. Phys. Solids, vol. 51, no. 8, pp. 1413-1431, 2003.

[15] G. Y. Zhang, M. L. Xu, R. Q. Zhang, and W. H. Tang, "Study of Stress Wave Propagation with NHDMOC," vol. 5, no. 1, pp. 781-785, 2011. 


\section{تعديل جهاز قياس الحمل الايناميكي هويكسنسون \\ مع الاخذ في الاعتبار حساب الترحيل الزمني للموجات}

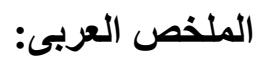

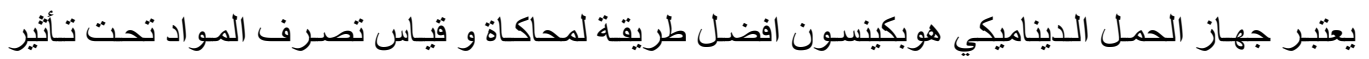

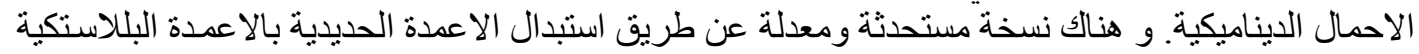

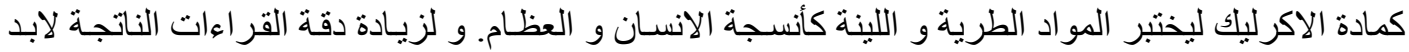

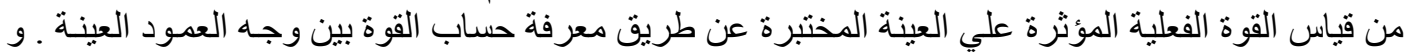

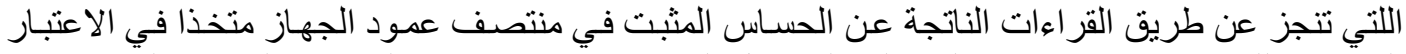

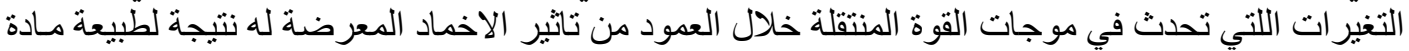

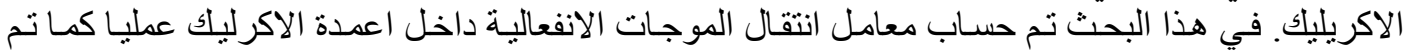
حساب معاملات اخماد الموجة و معامل التناقص في سر عة تللك الموجة. 